\title{
Laparoscopic Vaginal Suspension and Rectopexy for Rectal Prolapse
}

\author{
Shi-Jun Yang, Seo-Gue Yoon, Ki-Yun Lim, Jong-Kyun Lee \\ Department of Surgery, Seoul Song Do Hospital, Seoul, Korea
}

Purpose: Laparoscopic procedures for the treatment of patients with a rectal prolapse have gained increasing worldwide acceptance because they have lower recurrence and better functional outcome than perineal procedures. Nevertheless, ideal surgical methods are still not available. We propose a new surgical technique, laparoscopic vaginal suspension and rectopexy, for correcting a full-thickness rectal prolapse and/or middle-compartment prolapse. This study assessed the short-term outcomes for patients who underwent laparoscopic vaginal suspension and rectopexy.

Methods: Between April 2014 and April 2016, 69 female patients underwent laparoscopic vaginal suspension and rectopexy to correct a rectal prolapse. Demographics, medical histories, and surgical and follow-up details were collected from their medical records. In addition to the clinical outcome, we repeated defecation proctography and a questionnaire regarding functional results three months after surgery.

Results: No major morbidities or no mortalities occurred. The defecation proctography confirmed excellent anatomical result in all cases. Of 7 patients with combined middle-compartment prolapses, we observed good anatomical correction. During follow-up, full-thickness recurrence occurred in one patient. Preoperative fecal incontinence was improved significantly at 3 months (mean Wexner score: 12.35 vs. 7.71; mean FISI: 33.29 vs. 21.07; P $<0.001$ ). Analysis of responses to the fecal incontinence quality of life (FIQOL) questionnaire showed overall improvement at 3 months compared to the preoperative baseline (mean pre- and postoperative FIQOL scores: 12.11 vs. 14.39; $\mathrm{P}<0.004$ ).

Conclusion: Laparoscopic vaginal suspension and rectopexy is a new combined procedure for the treatment of patients with rectal prolapses. It has excellent functional outcomes and minimal morbidity and can correct and prevent middlecompartment prolapses.

Keywords: Rectal prolapse; Middle compartment prolapse; Ventral rectopexy; Pelvic organ prolapse suspension

\section{INTRODUCTION}

Rectal prolapse is a disease wherein the rectum is prolapsed to the anal canal because of full-thickness intussusception of the upper rectum, which may be internal or extrude externally. Rectal prolapse is more common in elderly women after the fifth decade [1]. Other pelvic-floor disorders in women may be present in $8 \%$ to

Received: September 26, 2016 • Accepted: February 26, 2017

Correspondence to: Seo-Gue Yoon, M.D.

Department of Surgery, Seoul Song Do Hospital, 72, Dasan-ro, Jung-gu, Seoul 04597, Korea

Tel: +82-2-2250-7368, Fax: +82-2-2233-8528, E-mail: seogue@naver.com

(c) 2017 The Korean Society of Coloproctology

This is an open-access article distributed under the terms of the Creative Commons Attribution NonCommercial License (http://creativecommons.org/licenses/by-nc/4.0) which permits unrestricted noncommercial use, distribution, and reproduction in any medium, provided the original work is properly cited.
$27 \%$ of patients with rectal prolapse. Weakness of the pelvic diaphragm and rectovaginal fascia allow descent of the middle compartment, which may present as a rectocele, enterocele, and vaginal vault prolapse [2].

The purpose of surgery for rectal prolapse is to correct the prolapse and to recover defecation dysfunction postoperatively. Simultaneous correction of associated middle-pelvic-compartment prolapses should be achieved, if possible. Numerous types of surgical procedures have been described, which include abdominal and perineal procedures. Abdominal procedures are techniques for fixation of the rectum to the sacral bone by using diverse methods, and excessive sigmoid colon or rectum may be resected in combination. Perineal procedures are techniques for shortening the prolapsed rectum by cutting or placating the prolapsed rectum. Surgery has evolved from a perineal to an abdominal approach, which can now be performed in a minimally-invasive 
manner [3]. Nevertheless, ideal surgical procedures are still not available.

Laparoscopic ventral rectopexy is the most recent commonlyused technique. Observational and retrospective studies show good functional results, low complication rates, and low recurrence rates [4]. This procedure is durable and minimally invasive; it spares the autonomic nerve and does not require a colonic resection. However, currently, concerns, such as erosion, sepsis, rectal stricture, pelvic pain or dyspareunia, and mesh detachment, exist over the synthetic polypropylene mesh located between the rectum and vagina $[5,6]$.

Ceci et al. [7]. published their article on pelvic organ prolapse suspension (POPS) in 2013. POPS is a recent surgical procedure for 1-stage treatment of a multiorgan female pelvic prolapse. This surgical procedure consists of fixing a $\mathrm{V}$-shaped prolene mesh to the apex of the anterior vaginal fornix and making a subperitoneal tunnel for both mesh strips. Patients undergo a stapled transanal rectal resection (STARR) to correct the residual rectal prolapse at the same time. POPS can achieve good anatomical results not only for vaginal prolapse, but also for rectocele, enterocele, and/or rectal prolapse, without functional impairments and complications.

Based on this background, we modified the POPS procedure and proposed a new combined surgical technique, laparoscopic vaginal suspension and rectopexy, for rectal prolapse correction. A rectal prolapse can be corrected completely by using this modified POPS procedure and three strips of polypropylene mesh; the STARR procedure is not needed. Correcting a middle-compartment prolapse may also be possible. The current study attempts to present short-term outcomes in patients who have undergone this new procedure.

\section{METHODS}

\section{Patients and procedures}

Female patients having an external rectal prolapse (Oxford classification grade $\mathrm{V}$ ) with or without a middle-compartment prolapse were selected for surgery, after women of child-bearing age or elderly patients with significant comorbidities had been excluded. Mesh-related morbidity is a concern in young women. The patients who could not support prolonged general anesthesia underwent a Delorme procedure. Between April 2014 and April 2016, 69 women patients underwent laparoscopic vaginal suspension and rectopexy for rectal prolapse correction at our hospital. Diagnosis of rectal prolapse was suggested based on history of symptoms and clinical examination findings and was confirmed radiologically. Clinically, all patients had a full-thickness external rectal prolapse that was confirmed by using defecation proctography. The patients underwent preoperative colonoscopy and magnetic resonance imaging to exclude organic disease. All patients also underwent anal manometry for an anorectal physiology evaluation. After signing the informed consent, patients were asked to complete a questionnaire regarding functional results. This questionnaire included the Cleveland Clinic Incontinence Score (CCIS, Wexner score), the Fecal Incontinence Severity Index (FISI), and the Fecal Incontinence Quality of Life (FIQOL) Scale $[8,9]$. Demographics, medical history, and surgical and follow-up details were collected from the patients' medical records. Follow-ups were planned for 2 weeks postoperatively and one follow-up at 3 months. We repeated defecation proctography and the questionnaire involving the CCIS, FISI, and FIQOL 3 months after the surgery.

\section{Laparoscopic surgical technique}

General anesthesia was necessary in all cases. We placed the patient in the lithotomy position with both arms near the body and with the thighs spread moderately and bent upwards. After appropriate preparation and draping, we inserted a Foley catheter. Laparoscopy was performed through 5 trocars: one $12-\mathrm{mm}$ balloon trocar in the umbilicus, one 5-mm trocar in the right upper quadrant, one 11-mm trocar in the right iliac fossa, one $5-\mathrm{mm}$ trocar in the left upper quadrant, and one 5-mm trocar in the left iliac fossa. The table was tilted in a marked Trendelenburg position to facilitate small-bowel abdominal retraction.

If present, the uterus was hitched to the anterior abdominal wall by using $2 / 0$ nylon on a straight needle. The sigmoid colon was retracted out of the pelvis and to the left side. An incision was made in the peritoneum over the right side of the sacral promontory. Medial dissection was extended over the sacral promontory along the sulcus toward the Douglas pouch. The peritoneum of the Douglas pouch was excised to be free of the anterior rectal muscular wall and the posterior vaginal vault. At the sacral promontory level, dissection was done close to the fascia propria of the rectum to avoid injury to the hypogastric nerves. The posterior dissection was brought down to the level of the coccyx. During lateral dissection, lateral ligaments containing rectal branches from the pelvic plexus were preserved.

With the use of polypropylene mesh (Optilene, B.Braun, Rubi, Spain), a trimmed 3-strip mesh (Fig. 1; 30-cm length and 2-cm width in each arm) was prepared. The mesh was introduced into

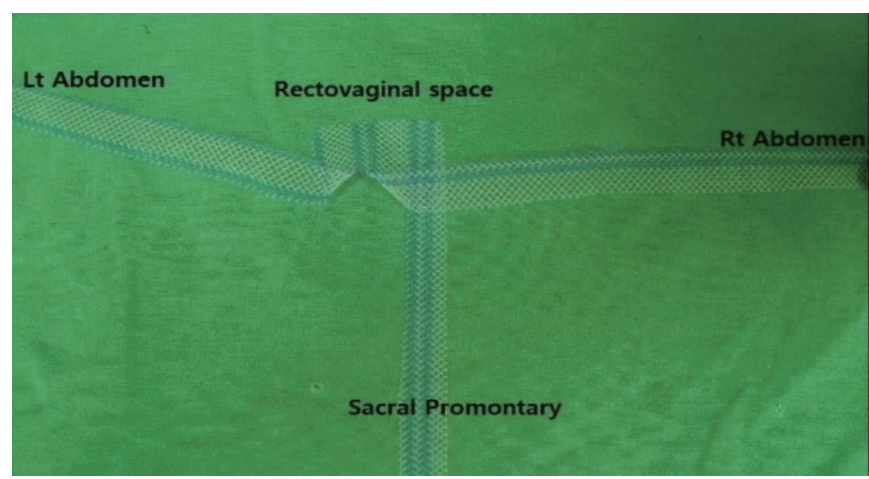

Fig. 1. Mesh configuration. LT, left; RT, right. 
the abdominal cavity through the 11-mm trocar. Under laparoscopic vision, the subperitoneal tunnel was performed, starting from the iliac crest trocar to $2 \mathrm{~cm}$ above the peritoneal reflection of the rectum. Both mesh ends were pulled out through the subperitoneal tunnel, and pelvic organ suspension was achieved by making symmetrical tractions on both mesh strips. The base of the polypropylene mesh was fixed by using a 2/0 Monosyn suture (B.Braun) on the posterior vaginal vault or on the vaginal apex if the patient had had a hysterectomy. A third strip of the mesh was fixed to the sacral promontory (Fig. 2). The rectum was lifted by the assistant, and a point on the sacral promontory was selected for the rectopexy.

After complete anatomic correction of the prolapse had been checked by traction of the rectum, the rectum was fixed to the sacral promontory by suturing with $2 / 0$ polypropylene (SM Eng, Busan, Korea) for rectopexy. In this procedure, synthetic mesh was sutured to the right posterior lateral mesorectum. We believe that fixation to the fatty mesorectum may be weak in the pull-out pressure. The mesh was then sutured to the mesorectal fascia with nonabsorbable sutures. The peritoneum was then closed over the mesh by using an absorbable continuous suture. A shallow and narrow new Douglas pouch was created by using a peritoneum closure over the mesh. A 5-cm excess mesh strip was positioned, tunneling the muscle's fascia, above the incision and fixed by vicryl 2/0 sutures. The skin was sutured with an intradermal suture.

\section{Statistical analysis}

All statistical analyses were performed using IBM SPSS ver. 22.0 (IBM Co., Armonk, NY, USA). Quantitative data were expressed as medians and ranges. Analyses were performed using the paired $\mathrm{t}$-test for paired data. We considered P-values less than 0.05 to be statistically significant.

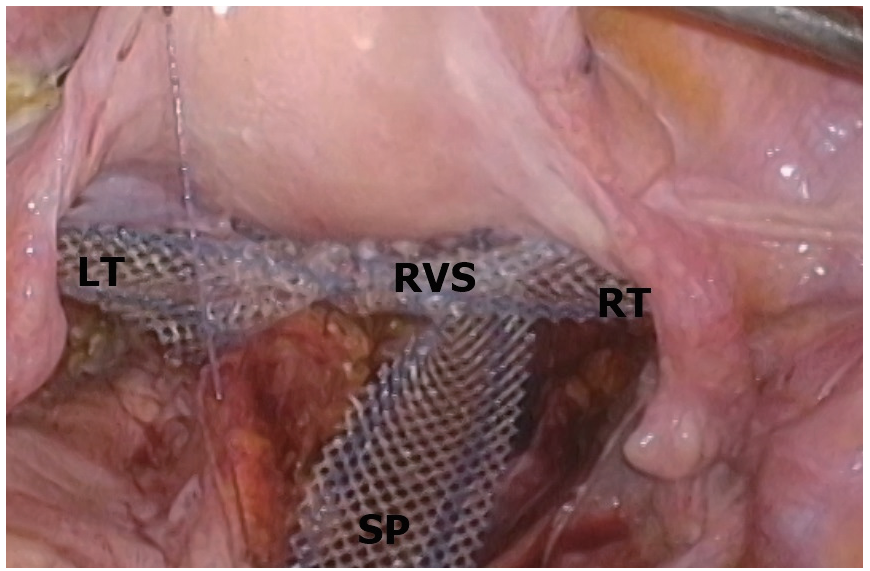

Fig. 2. The base of the mesh is fixed to the posterior vaginal fornix. Symmetrical tractions on both mesh strips reduce the rectal prolapse, middle-compartment prolapse. A third strip of the mesh was fixed to the sacral promontory. RVS, rectovaginal space; SP, sacral promontory; LT, left; RT, right.

\section{RESULTS}

Between April 2014 and April 2016, 69 women with a median age of 72 years (range, 50-95 years) and median body mass index of $23.91 \mathrm{~kg} / \mathrm{m}^{2}$ (range, 16.36-33.32 kg/m²) underwent laparoscopic vaginal suspension and rectopexy for full-thickness prolapse. Forty-eight patients were multiparous. The median parity was 3 (range, 0-8). Thirty-nine percent of the patients had undergone previous pelvic floor surgery. Recurrent rectal prolapse was presented in 19 patients after previous rectal prolapse surgery. Of these, 16 underwent Delorme's procedure, 1 an Altemeier procedure, and 2 an abdominal rectopexy. A hysterectomy had been previously performed in 11 patients. The most frequent complaint at the time of clinical presentation was the sensation of a protruding rectal mass $(67,97 \%)$. Additional symptoms and clinical findings included fecal incontinence (16, 23\%), constipation $(7,10 \%)$, rectal bleeding $(11,16 \%)$, and anal pain $(7,10 \%)$. Using preoperative defecation proctography, we found a rectocele in 21 patients. A uterine prolapse and a vaginal vault prolapse were detected in three and four patients, respectively.

The operative data for the 69 patients are shown in Table 1. No conversion was necessary, and no intraoperative complications were observed. No postoperative mortality was found. Postoperative complications were minimal ( 2 port-site infections, 1 woundsite bleeding). No patient was readmitted for surgical complications secondary to the laparoscopic vaginal suspension and rectopexy. No mesh-related complications were found. One recurrent full-thickness rectal prolapse occurred 5 months postoperatively. We recommended a Delorme's procedure to that patient. However, the patient was lost to follow-up. Two patients complained of recurrent mucosal prolapse, which was treated successfully by using rubber-band ligation. Follow-up was planned 2 weeks postop-

Table 1. Operative data

\begin{tabular}{lc}
\hline Variable & Value \\
\hline ASA PS classification & \\
\hline I & $4(5.8)$ \\
III & $58(84.1)$ \\
Operation time (min) & $7(10.1)$ \\
Postoperative complication & $105(60-150)$ \\
Wound infection & \\
Wound hematoma & 2 \\
Hospital stay (day) & 1 \\
Recurrence & $7(4-11)$ \\
Full-thickness prolapse & \\
Rectal mucosal prolapse & 1 \\
\hline
\end{tabular}

Values are presented as number (\%), median (range), or number. ASA PS, American Society of Anesthesiologists physical status. 
Table 2. Changes in the incontinence functional data and anal sphincter function after surgery

\begin{tabular}{lccr}
\hline Variable & Preoperative & Postoperative & P-value \\
\hline Mean Wexner score & 12.35 & 7.71 & $<0.001$ \\
Mean FISI score & 33.29 & 21.07 & $<0.001$ \\
Mean FIQOL score & 12.11 & 14.39 & 0.004 \\
Maximum resting pressure $(\mathrm{mmHg})$ & 27.8 & 31.34 & 0.007 \\
Maximum squeezing pressure $(\mathrm{mmHg})$ & 66.2 & 70.1 & 0.183 \\
\hline
\end{tabular}

FISI, Fecal Incontinence Severity Index; FIQOL, fecal incontinence quality of life.

eratively and 3 months thereafter. We repeated defecation proctography 3 months after the surgery. Defecation proctography confirmed excellent anatomical result in all cases. We also observed good anatomical correction of the vaginal vault prolapse or uterine prolapse in the 7 patients with combined middle-compartment prolapse.

Incontinence functional data were complete in 38 of the $69 \mathrm{pa}-$ tients (55.1\%). Functional assessments were impossible for $31 \mathrm{pa}-$ tients. At the 3-month follow-up, the patients showed notable improvements in fecal incontinence and quality of life after surgery. The changes in the functional data are shown in Table 2. The Wexner fecal incontinence and FISI scores were significantly reduced at the 3-month follow-up. Analysis of responses on the FIQOL questionnaire showed overall improvements at the 3-month follow-up compared with the preoperative baseline. With regard to the anal sphincter function, the anal maximum resting pressures were slightly increased, with statistical significance, after surgery.

\section{DISCUSSION}

Complete rectal prolapse is a lifestyle-altering disability that commonly affects older people. The only potentially curative treatment for full-thickness rectal prolapse is surgery. Many surgical methods have been introduced and are described in the literature. However, ideal surgical methods are still not available. The range of surgical methods available to correct the underlying pelvic floor defects in full-thickness rectal prolapse reflects the lack of consensus regarding the best surgery. Two predominant general approaches, abdominal and perineal, are considered in the surgical repair of a complete rectal prolapse [10]. Perineal resections have a lower operative risk and morbidity. They may, therefore, be more suitable for older or high-risk patients with a relevant comorbidity [11]. Abdominal surgeries have been generally accepted to carry a lower recurrence rate and improved functional outcome and are, therefore, preferred over perineal surgeries [12].

Abdominal procedures for rectal prolapse play a major role in the treatment. Numerous abdominal approaches have been introduced. Surgical techniques may consist of mobilization and resection of the rectosigmoid colon, fixation of the rectum to the sa- crum, or a combination of these procedures. Virtually all abdominal procedures that were originally described via laparotomy can also be performed laparoscopically. The laparoscopic management of a rectal prolapse was first introduced in 1992 and consisted of a suture-less rectopexy with staples without bowel resection [13]. Laparoscopic surgery has the advantages of less pain, earlier return to diet, short hospital stay, and early return to work [14].

Laparoscopic ventral rectopexy has gained increasing worldwide acceptance. In 2004, D’Hoore et al. [15] first described ventral rectopexy repair and its potential advantage in avoiding postoperative constipation. This is accomplished by avoiding dissection to the posterior and lateral aspects of the rectum, thereby sparing the autonomic nerve [16]. Unlike a posterior rectopexy, a ventral rectopexy has the potential to correct any coexisting middle-compartment prolapse. A systematic review by Samaranayake et al. [17] indicated that the recurrence rates of ventral rectopexy were low, ranging from $0 \%$ to $15.4 \%$. A laparoscopic ventral rectopexy is a novel development in the treatment of rectal prolapse. However, the theoretically-limited anterior rectal dissection means that this approach can only achieve anterior rectal fixation. Hence, a portion of the failure/recurrence rate is speculated to be due to the posterior residual prolapse in patients who undergo a laparoscopic ventral rectopexy. Although mesh-related complications are rare in the literature [18] - 1 case of erosion of the posterior vaginal wall and 2 cases of mesh detachment, real concerns regarding permanent mesh remain. Because the mesh is applied deeply between the rectum and vagina in a ventral rectopexy, risks exist for infection, erosion into the rectum or vagina, rectal stricture, rectovaginal fistula, and chronic pelvic pain [19].

In 2013, Ceci et al. [7] evaluated the preliminary results on laparoscopic POPS. POPS is a recent surgical procedure for 1-stage treatment of a multiorgan female pelvic prolapse. This surgical procedure consists of fixing a $\mathrm{V}$-shaped prolene mesh to the apex of the anterior vaginal fornix and making a subperitoneal tunnel for both mesh strips. Pelvic organ suspension is achieved by making symmetrical tractions on both mesh strips. Finally, each mesh strip end is fixed to the abdominal muscle fascia. POPS can achieve good anatomical results not for only vaginal prolapse but also for rectocele, enterocele, and rectal prolapse, without functional impairments and complications (mesh-related erosion of the rectum or dyspareunia). However, POPS may be associated with a persisting and recurring rectal prolapse. If a residual rectal prolapse persists, the STARR procedure is performed.

Based on this background, we modified the POPS procedure and introduced a combined technique for correcting a rectal prolapse, laparoscopic vaginal suspension and rectopexy. Instead of using a V-shaped polypropylene mesh with 2 strips in POPS, we trim the polypropylene mesh into a 3-strip mesh. The mesh is sutured to the posterior vaginal fornix, and lateral suspension is achieved similarly to POPS. This procedure can correct and prevent a middle-compartment prolapse. Finally, a third strip of the 
mesh is fixed upon the sacral promontory with the mobilized rectal wall. Because of rectum fixation to the third mesh strip, the rectal prolapse is corrected completely, and the STARR procedure is not needed. Unlike in a ventral rectopexy, the rectum is mobilized anteriorly and posteriorly, but with limited dissection, not beyond the lateral ligament, to preserve the nerve supply to the rectum. This is a posterior mesh fixation with the addition of pelvic organ suspension, which can elevate the remaining circumferential elements of the prolapse indirectly

We obtained promising postoperative functional outcomes and good defecographic findings. Regarding the effect on continence, significant improvement was found. The position of the mesh makes the procedure safe from mesh erosion or infection and dyspareunia. Furthermore, the fixation of the vaginal vault on the same mesh corrects the prolapse of the middle pelvic compartment. In our study, 1 patient had a recurrence of the rectal prolapse at 5 months. No obvious factors associated with recurrence were found. Regarding the operative technique, we have routinely used a 2/0 absorbable suture (PDS II, polydioxanone) to secure the mesh during early experience. We suspected that using an absorbable stitch contributed to recurrence, so we changed the absorbable suture to a non-absorbable suture.

This study has several limitations. Of 69 patients with rectal prolapse, symptoms of obstructed defecation were resolved in 5 of 7 patients. During follow-up, new onsets of mild obstructed defecation were noted in 15 patients. However, we did not routinely obtain the constipation questionnaire score during the evaluation. A comparison of validated scores before and after surgery was, consequently, impossible. We assumed that rectal wall edema secondary to the rectal mobilization contributed to this phenomenon. Structural change mechanisms could alter the passage of stool to the lower rectum. Hence, we should collect follow-up data for functional constipation results. Although mesh complications are rare among the published series of POPS, the long-term effects of permanent mesh in the pelvis are unknown. Our follow-up duration was relatively short, and real concerns regarding permanent mesh exist. This study was limited because the patients were followed short term. We should develop a follow-up protocol that is sufficiently long and collect data from many case studies to support our claims. Nevertheless, we believe laparoscopic vaginal suspension and rectopexy to be an effective procedure for the correction of a rectal prolapse and/or a middle-compartment prolapse.

In conclusion, laparoscopic vaginal suspension and rectopexy is a new combined procedure with excellent functional outcomes and minimal morbidity. It allows a short hospital stay and alleviates symptoms of incontinence. The advantages of the technique are its low recurrence rate and the absences of both mesh-related complications and postoperative functional impairment. In addition, it can be used to correct and prevent middle-compartment prolapses. Follow-up on these patients is necessary to achieve long-term outcomes.

\section{CONFLICT OF INTEREST}

No potential conflict of interest relevant to this article was reported.

\section{REFERENCES}

1. Kairaluoma MV, Kellokumpu IH. Epidemiologic aspects of complete rectal prolapse. Scand J Surg 2005;94:207-10.

2. González-Argenté FX, Jain A, Nogueras JJ, Davila GW, Weiss EG, Wexner SD. Prevalence and severity of urinary incontinence and pelvic genital prolapse in females with anal incontinence or rectal prolapse. Dis Colon Rectum 2001;44:920-6.

3. Yoon SG, KIm KU, Noh KY, Lee JK, Kim KY. Laparoscopic suture rectopexy for rectal prolapse. J Korean Soc Coloproctol 2002;18: 89-94.

4. Rickert A, Kienle P. Laparoscopic surgery for rectal prolapse and pelvic floor disorders. World J Gastrointest Endosc 2015;7:104554.

5. Adeyemo D. Mesh fistulation into the rectum after laparoscopic ventral mesh rectopexy. Int J Surg Case Rep 2014;5:152-4.

6. Tranchart H, Valverde A, Goasguen N, Gravié JF, Mosnier H. Conservative treatment of intrarectal mesh migration after ventral laparoscopic rectopexy for rectal prolapse. Int J Colorectal Dis 2013;28:1563-6.

7. Ceci F, Spaziani E, Corelli S, Casciaro G, Martellucci A, Costantino A, et al. Technique and outcomes about a new laparoscopic procedure: the Pelvic Organ Prolapse Suspension (POPS). G Chir 2013;34:141-4.

8. Rockwood TH, Church JM, Fleshman JW, Kane RL, Mavrantonis $\mathrm{C}$, Thorson AG, et al. Patient and surgeon ranking of the severity of symptoms associated with fecal incontinence: the fecal incontinence severity index. Dis Colon Rectum 1999;42:1525-32.

9. Rockwood TH, Church JM, Fleshman JW, Kane RL, Mavrantonis C, Thorson AG, et al. Fecal Incontinence Quality of Life Scale: quality of life instrument for patients with fecal incontinence. Dis Colon Rectum 2000;43:9-16.

10. Varma M, Rafferty J, Buie WD; Standards practice task force of American Society of Colon and Rectal Surgeons. Practice parameters for the management of rectal prolapse. Dis Colon Rectum 2011;54:1339-46.

11. Hammond K, Beck DE, Margolin DA, Whitlow CB, Timmcke AE, Hicks TC. Rectal prolapse: a 10-year experience. Ochsner J 2007;7:24-32.

12. Deen KI, Grant E, Billingham C, Keighley MR. Abdominal resection rectopexy with pelvic floor repair versus perineal rectosigmoidectomy and pelvic floor repair for full-thickness rectal prolapse. Br J Surg 1994;81:302-4.

13. Berman IR. Sutureless laparoscopic rectopexy for procidentia. Technique and implications. Dis Colon Rectum 1992;35:689-93.

14. Kellokumpu IH, Vironen J, Scheinin T. Laparoscopic repair of rectal prolapse: a prospective study evaluating surgical outcome 
and changes in symptoms and bowel function. Surg Endosc 2000; 14:634-40.

15. D'Hoore A, Cadoni R, Penninckx F. Long-term outcome of laparoscopic ventral rectopexy for total rectal prolapse. Br J Surg 2004; 91:1500-5.

16. Lauretta A, Bellomo RE, Galanti F, Tonizzo CA, Infantino A. Laparoscopic low ventral rectocolpopexy (LLVR) for rectal and rectogenital prolapse: surgical technique and functional results. Tech Coloproctol 2012;16:477-83.

17. Samaranayake CB, Luo C, Plank AW, Merrie AE, Plank LD, Bis- sett IP. Systematic review on ventral rectopexy for rectal prolapse and intussusception. Colorectal Dis 2010;12:504-12.

18. Faucheron JL, Voirin D, Riboud R, Waroquet PA, Noel J. Laparoscopic anterior rectopexy to the promontory for full-thickness rectal prolapse in 175 consecutive patients: short- and long-term follow-up. Dis Colon Rectum 2012;55:660-5.

19. Badrek-Al Amoudi AH, Greenslade GL, Dixon AR. How to deal with complications after laparoscopic ventral mesh rectopexy: lessons learnt from a tertiary referral centre. Colorectal Dis 2013;15: 707-12. 